\title{
A principal-components-based clustering method to identify multiple variants associated with rheumatoid arthritis and arthritis-related autoantibodies Mary Helen Black and Richard M Watanabe*
}

Address: Department of Preventive Medicine, Keck School of Medicine, University of Southern California, 1540 Alcazar Street, CHP 222-V, Los Angeles, California 90089, USA

E-mail: Mary Helen Black - mhbridge@usc.edu; Richard M Watanabe* - rwatanab@usc.edu

*Corresponding author

from Genetic Analysis Workshop 16

St Louis, MO, USA 17-20 September 2009

Published: 15 December 2009

BMC Proceedings 2009, 3(Suppl 7):SI29 doi: 10.1I86/I753-656I-3-S7-SI29

This article is available from: http://www.biomedcentral.com/I753-656I/3/S7/SI29

(C) 2009 Black and Watanabe; licensee BioMed Central Ltd.

This is an open access article distributed under the terms of the Creative Commons Attribution License (http://creativecommons.org/licenses/by/2.0), which permits unrestricted use, distribution, and reproduction in any medium, provided the original work is properly cited.

\begin{abstract}
Multivariate techniques are an important area of investigation for studying contributions of multiple genetic variants to disease onset and pathology. We analyzed the Genetic Analysis Workshop 16 North American Rheumatoid Arthritis Consortium (NARAC) data using a principal-components analysis (PCA) with an orthoblique rotation to identify specific subsets of single-nucleotide polymorphisms (SNP) in the major histocompatibility complex (MHC) region associated with rheumatoid arthritis (RA) and rheumatoid factor IgM (RFUW), and compared this method with a traditional PC approach. Using the orthoblique PC-based clustering method, we identified new clusters of SNPs across the MHC region associated with RA and RFUW, and replicated known SNP cluster associations with RA, such as those in the HLA-DRB region.
\end{abstract}

\section{Introduction}

Testing a candidate gene or region for association with phenotypes typically involves testing multiple singlenucleotide polymorphisms (SNPs). This necessarily introduces the issue of multiple test corrections, which reduce power in order to control the type 1 error rate. Therefore, development of multivariate methods to identify causal loci and to reduce the burden of multiple testing is an area of ongoing investigation for complex diseases. Several multivariate techniques have been used to examine whether multiple SNPs are associated with disease or quantitative traits [1-4]. However, such methods typically suffer from low power under various scenarios or the inability to reduce the large number of SNPs to a smaller subset that may point to a specific location within the region.

Gauderman et al. introduced a principal-components method to assess whether multiple variants within a candidate gene are associated with disease [3]. Principalcomponents analysis (PCA) is used to derive linear transformations of the original SNP data, in which eigenvectors are chosen to maximize the variance of each PC relative to the overall variation in the region [3]. Each 
eigenvalue represents the variance of a particular PC, and typically, only a subset of PCs that account for a large proportion of the total variation are chosen for analysis, reducing the number of parameters to be tested. These PCs serve as covariates in an omnibus test of association with disease or trait $[3,4]$.

The PC approach has been shown to have greater power than standard joint SNP or haplotype-based tests to detect association between multiple SNPs and disease, especially when the number of haplotypes is large [3]. However, the coefficients of each eigenvector are derived from pair-wise correlations among the SNPs, and thus lack specific interpretation. Eigenvector loadings of the original variables on a PC do not reflect the true importance of the SNPs to that PC, making the association between multiple PCs and disease outcomes difficult to interpret [4].

We propose a PC-based clustering method as an alternative approach that reduces dimensionality of the data and maintains the power of a PC approach, but allows for unique identification of multiple SNPs in the region being tested. The algorithm uses an orthoblique rotation of PCs on genotype data to form distinct clusters, where each cluster is defined by a specific array of SNPs. A subset of clusters that explains a large proportion of the total locus variation is selected, such that those clusters can be tested for association with disease outcomes.

The PC approach and our proposed oblique PC-based clustering method were applied to the analysis of rheumatoid arthritis (RA) data from Genetic Analysis Workshop 16 (GAW16) (Problem 1). We compare and contrast results from these two approaches and compare findings with previously published results for these data [5-8].

\section{Methods}

\section{Sample data}

Sample data included genome-wide association data from Affymetrix GeneChip $100 \mathrm{k}$ Mapping Array containing 116,204 SNPs for RA and RA-related traits, such as rheumatoid factor IgM (RFUW) and anti-cyclic citrullinated peptide (anti-CCP), on 2,062 North American Rheumatoid Arthritis Consortium (NARAC) subjects. Of the 1,250 SNPs in the major histocompatibility complex (MHC) region on 6p21 spanning 3.2 Mb, we restricted analysis to individuals with RA status and complete genotype data ( $n=1,187$ on 838 SNPs).

\section{Pre-analysis processing of data}

Observed genotype frequencies were assessed for deviation from Hardy-Weinberg equilibrium and allele frequencies estimated using the computer program Haploview (V.4.1). For these analyses, we excluded markers with minor allele frequencies $(\mathrm{MAFs})<0.01$, and coded genotypes as 0,1 , or 2 according to the number of minor alleles. Log transforms were applied to quantitative trait data to approximate univariate normality. We performed PCA as described by Gauderman et al. [3]. The subset $s$ of PCs used in the analysis was determined by the quantity that accounted for $80 \%$ of the total variation.

The PC-based clustering method begins with the total $k$ SNPs initially grouped into a single cluster. Standard PCA is performed on the initial cluster, with an orthoblique rotation [9] of the first two PCs $\left(\mathrm{PC}_{1}\right.$, $\mathrm{PC}_{2}$ ). Each SNP is assigned to the rotated component with which it has the higher squared correlation, dividing the initial cluster into two disjointed clusters. PC analysis within newly formed clusters and SNP assignment continue iteratively, assigning SNPs to clusters, and then re-testing each SNP to determine whether assigning it to a different cluster increases the amount of variance explained, with the goal of maximizing the total variance accounted for by the cluster components. For $k$ SNPs, we computed $n$ clusters:

$$
\begin{gathered}
\mathrm{C}_{1}=\mathbf{c}_{\mathbf{1}} \mathbf{g}=c_{11} g_{1}+c_{21} g_{2}+\ldots+c_{k 1} g_{k} \\
\vdots \\
\mathrm{C}_{n}=\mathbf{c}_{\mathbf{n}} \mathbf{g}=c_{1 n} g_{1}+c_{2 n} g_{2}+\ldots+c_{k n} g_{k,}
\end{gathered}
$$

where $C_{n}$ is the $n^{\text {th }}$ cluster score, $\mathbf{c}_{\mathbf{n}}$ is a vector of standardized cluster coefficients, and $\mathrm{g}$ is the vector of SNPs $\left[g_{1}, g_{2}, \ldots, g_{k}\right]$. While all SNPs are subdivided into a total of $n$ clusters, the number of SNPs within each cluster varies, yielding cluster coefficients equal to zero for SNPs not included in the $n^{\text {th }}$ cluster. Given the cluster coefficients and individual genotype scores, cluster scores are computed for each individual. For comparison with traditional PC analysis, $n$ was determined by the number of clusters that accounted for $80 \%$ of the total locus variation.

\section{Association testing}

For disease status, we analyzed PC scores $\left(\mathrm{PC}_{s}\right)$ or cluster score $\left(\mathrm{C}_{n}\right)$, using the following logistic framework:

$$
\operatorname{Logit}\left[\operatorname{Pr}\left(D=1 \mid C_{1}, \ldots, C_{n}\right)\right]=\beta_{0}+\beta_{1} C_{1}+\ldots+\beta_{n} C_{n} .
$$

For continuous outcomes, we fit a multiple linear regression model with PC scores or cluster scores as covariates. Likelihood-ratio tests were used to contrast the null model (intercept only) to that with either $s$ PCs (if traditional PCA) or $n$ clusters (if cluster analysis) to assess significance, with $s$ or $n$ degrees of freedom (df). Given significant association under omnibus test of all 
PCs or PC-based clusters, 1-df Wald tests were used to test association between RA or RA-related trait with each PC or PC-based cluster conditional upon all PCs or clusters. Because PC and cluster scores are estimated from the correlation structure of the genotype data, it should be noted that $p$-values resulting from any association testing framework may not be completely accurate. A bootstrap or randomization procedure that includes computation of PC scores or cluster components would likely yield more accurate $p$-values. For the purposes of this paper, nominal $p$-values are reported. All analyses were performed using SAS (v.9.1).

\section{Results}

Demographic data for 1,187 subjects (331 male, 856 female) included in this analysis are shown in Table 1. Data analyzed consisted of 515 cases, 672 controls. RArelated traits were measured in cases only. Clinically relevant titres of anti-CCP $(\geq 25 \mathrm{U} / \mathrm{ml})$ were found in $98 \%$ of cases; for RFUW ( $\geq 20 \mathrm{IU} / \mathrm{ml}$ ), it was $95 \%$. Anti-CCP ranged from 20 to $2,554 \mathrm{U} / \mathrm{ml}$, with a mean \pm SD of 194.9 $\pm 234.9 \mathrm{U} / \mathrm{ml}$. RFUW ranged from 9 to $4,225 \mathrm{IU} / \mathrm{ml}$, with mean \pm SD $271.0 \pm 471.7 \mathrm{IU} / \mathrm{ml}$

Of the 838 SNPs in the MHC region, nine were monomorphic and seven had MAFs $<0.01$. PC analysis yielded 53 PCs that accounted for $80 \%$ of the variance and were used for association testing. The MHC region was significantly associated with RA status $\left(p=4.8 \times 10^{-85}\right)$ and RFUW ( $p=0.0159$ ) (Table 2). We found no evidence for association with PCs and anti-CCP $(p=0.62)$. Of the 53 PCs tested for association, 29 individual PCs were significantly associated with RA $(p \leq 0.05)$ and 8 were significantly associated with RFUW ( $p \leq 0.05) ; 5$ PCs associated with both RA and RFUW. Among all PCs

Table I: Subject characteristics

\begin{tabular}{lcc}
\hline & $\begin{array}{c}\text { Cases } \\
(\boldsymbol{n}=\mathbf{5 1 5})\end{array}$ & $\begin{array}{c}\text { Controls } \\
(\boldsymbol{n}=\mathbf{6 7 2})\end{array}$ \\
\hline Sex & & \\
$\quad$ Male $n(\%)$ & $131(25.4 \%)$ & $200(29.8 \%)$ \\
Female $n(\%)$ & $384(74.6 \%)$ & $472(70.2 \%)$ \\
Anti-CCP (units/mL) Mean (SD) & $194.9(234.9)$ & N/A \\
RFUW (IU/mL) Mean (SD) & $271.1(471.7)$ & N/A \\
\hline
\end{tabular}

significantly associated with either RA or RFUW, the eigenvector coefficients ranged from -0.1480 to 0.1453 . Therefore, this analysis allows us to conclude that the MHC region is associated with both RA and RFUW, but does not permit us to distinguish the relative contribution of each of the 822 SNPs to this association.

Our PC-based clustering algorithm identified 188 clusters that accounted for $80 \%$ of the variance and were used to test for association. Cluster size ranged from 1 to 14 SNPs. Using likelihood-ratio tests, the PCbased cluster method also found significant association between the MHC region and RA $\left(p=1.4 \times 10^{-74}\right)$ and RFUW $\left(p=5.7 \times 10^{-7}\right)$ (Table 2$)$. Similar to the PC analysis, the PC-based clustering method showed no evidence for association between the MHC region and anti-CCP $(p=0.21)$. Twenty-four SNP clusters were associated with RA $(p \leq 0.05)$ and 36 SNP clusters showed association with RFUW ( $p \leq 0.05)$; 2 clusters were common to both outcomes.

The two SNP clusters most significantly associated with RA are shown in Table 3. The majority of the SNPs in Cluster 1 are located in the MHC class I region, surrounding HLA-C and HLA-B. The squared correlation coefficients between each SNP and its assigned cluster $\left(\mathrm{R}_{\mathrm{O}}{ }^{2}\right)$, shown in Table 3, indicate that most SNPs in Cluster 1 share a high degree of correlation, and relatively low correlation with SNPs in any other cluster $\left(\mathrm{R}_{\mathrm{N}}{ }^{2}\right)$. Additionally, low values for the ratio of one minus each of these correlations indicates relatively stable SNP-cluster assignment. The SNPs in Cluster 23 primarily span $\sim 295$-kb region of MHC class II bounded by HLA-B and HLA-DQA1. The majority of these SNPs cluster around the HLA-DRA, HLA-DRB5, and HLA-DRB1 loci, are highly correlated with each other, and show stable SNP-cluster assignment.

The five SNP clusters most significantly associated with RFUW are shown in Table 4. The majority of the SNPs that comprise Clusters 2, 5, 24, and 183 are located in the MHC class 1 region. Clusters 2, 24, and 183 represent sets of SNPs between HLA-A and HLA-C, while SNPs in Cluster 5 span a $74-\mathrm{kb}$ region between HLA-C and $H L A-B$. Because the SNPs in Cluster 2 reside proximally

Table 2: HLA region association with rheumatoid arthritis and arthritis-related traits

\begin{tabular}{|c|c|c|c|c|c|c|c|}
\hline \multirow[b]{2}{*}{ Method } & \multirow[b]{2}{*}{ df } & \multicolumn{2}{|c|}{ RA affection status } & \multicolumn{2}{|c|}{ Anti-CCP } & \multicolumn{2}{|c|}{ RFUW } \\
\hline & & $\chi^{2}$ Statistic $^{a}$ & $p$-Value & $\chi^{2}$ Statistic $^{\mathrm{a}}$ & $p$-Value & $\chi^{2}$ Statistic ${ }^{a}$ & $p$-Value \\
\hline Traditional PC analysis & 53 & 556.274 & $4.8 \times 10^{-85}$ & 49.28 & 0.6197 & 77.432 & 0.0159 \\
\hline PC-based cluster analysis & 188 & 789.007 & $1.4 \times 10^{-74}$ & 203.383 & 0.2098 & 297.838 & $5.7 \times 10^{-7}$ \\
\hline
\end{tabular}

${ }^{a} \chi^{2}$ statistic based on multi-df likelihood ratio test for number of PCs or clusters included in the full model vs. the null model (intercept only). 
Table 3: Cluster associations with rheumatoid arthritis case-control status

\begin{tabular}{|c|c|c|c|c|c|c|c|}
\hline Cluster No. & SNP & Position $^{\mathrm{a}}$ & MAF & $\mathbf{R}_{\mathbf{O}}{ }^{2 \mathbf{b}}$ & $\mathbf{R}_{\mathbf{N}}^{2 \mathbf{c}}$ & $\left(I-\mathbf{R}_{\mathbf{O}}{ }^{2}\right) /\left(I-\mathbf{R}_{\mathbf{N}}{ }^{2}\right)$ Ratio & $p-$ Value $^{d}$ \\
\hline \multirow[t]{9}{*}{ I } & rs3130544 & $31,166,319$ & 0.125 & 0.805 & 0.682 & 0.612 & 0.0002 \\
\hline & rs2442749 & $31,460,019$ & 0.288 & 0.346 & 0.231 & 0.850 & \\
\hline & rs3099844 & $31,556,955$ & 0.113 & 0.897 & 0.570 & 0.240 & \\
\hline & rs9267444 & $31,591,437$ & 0.328 & 0.332 & 0.193 & 0.828 & \\
\hline & rs 2734583 & $31,613,459$ & 0.110 & 0.917 & 0.565 & 0.192 & \\
\hline & rs2857595 & $31,676,448$ & 0.165 & 0.648 & 0.334 & 0.529 & \\
\hline & rs3131379 & $31,829,012$ & 0.097 & 0.906 & 0.660 & 0.277 & \\
\hline & rs|270942 & $32,026,839$ & 0.096 & 0.885 & 0.697 & $0.38 I$ & \\
\hline & rs389884 & $32,048,876$ & 0.094 & 0.878 & 0.707 & 0.415 & \\
\hline \multirow[t]{10}{*}{23} & rs691007I & $32,390,832$ & 0.350 & 0.675 & 0.504 & 0.656 & 0.0008 \\
\hline & rs3817963 & $32,476,065$ & 0.402 & 0.880 & 0.558 & 0.271 & \\
\hline & rs3806I56 & $32,481,676$ & 0.453 & 0.787 & 0.534 & 0.458 & \\
\hline & rs3763309 & $32,483,951$ & 0.358 & 0.898 & 0.566 & 0.235 & \\
\hline & rs2395163 & $32,495,787$ & 0.355 & 0.901 & 0.574 & 0.232 & \\
\hline & rs2395I75 & $32,5 \mid 3,004$ & 0.312 & 0.841 & 0.485 & 0.308 & \\
\hline & rs2395185 & $32,541,145$ & 0.444 & 0.773 & 0.446 & $0.41 \mathrm{I}$ & \\
\hline & rs2516049 & $32,678,378$ & 0.429 & 0.825 & 0.423 & 0.303 & \\
\hline & rs660895 & $32,685,358$ & 0.361 & 0.832 & 0.515 & 0.346 & \\
\hline & rs532098 & $32,686,030$ & 0.489 & 0.701 & 0.312 & 0.434 & \\
\hline
\end{tabular}

aBased on HapMap Data Release 23a (Phase II), NCBI Build 36, dbSNP Build I 26.

${ }^{b}$ Squared correlation coefficient between a given SNP and its own cluster.

${ }^{\mathrm{c}}$ The next highest squared correlation coefficient between a given SNP and any other cluster.

${ }^{d} p$-Value from I df Wald $\chi^{2}$ for association with outcome, adjusted for all other clusters in the model.

Table 4: Cluster associations with RFUW

\begin{tabular}{|c|c|c|c|c|c|c|c|}
\hline Cluster No. & SNP & Position $^{a}$ & MAF & $\mathbf{R}_{\mathbf{O}}^{2 \mathbf{b}}$ & $\mathbf{R}_{\mathbf{N}}^{2 c}$ & $\left(I-\mathbf{R}_{\mathrm{O}}{ }^{2}\right) /\left(I-\mathbf{R}_{\mathbf{N}}{ }^{2}\right)$ Ratio & $p-$ Value $^{d}$ \\
\hline \multirow[t]{6}{*}{2} & rs2844670 & $31,113,705$ & 0.152 & $0.81 \mathrm{I}$ & 0.488 & 0.370 & 0.0004 \\
\hline & rs3130933 & $31,240,064$ & 0.133 & 0.945 & 0.522 & 0.116 & \\
\hline & rs3094609 & $31,273,545$ & 0.145 & 0.954 & 0.542 & 0.101 & \\
\hline & rs3130532 & $31,316,432$ & 0.142 & 0.956 & 0.531 & 0.094 & \\
\hline & rs7382297 & $31,355,046$ & 0.142 & 0.953 & 0.530 & 0.100 & \\
\hline & rs2905722 & $31,557,306$ & 0.139 & 0.747 & 0.614 & 0.656 & \\
\hline \multirow[t]{6}{*}{5} & rsII967684 & $31,307,745$ & 0.353 & 0.822 & 0.628 & 0.477 & 0.0004 \\
\hline & rs9468925 & $31,366,816$ & 0.353 & 0.902 & 0.464 & 0.183 & \\
\hline & rs3873379 & $31,370,148$ & 0.290 & 0.876 & 0.478 & 0.237 & \\
\hline & rs3873380 & $31,370,417$ & 0.303 & 0.938 & 0.499 & 0.124 & \\
\hline & rs9366778 & $31,377,152$ & 0.387 & 0.750 & 0.517 & 0.518 & \\
\hline & rs3873386 & $31,381,724$ & 0.374 & 0.852 & 0.514 & 0.305 & \\
\hline \multirow[t]{9}{*}{20} & rs|2177980 & $32,794,062$ & 0.447 & 0.888 & 0.443 & 0.202 & 0.0003 \\
\hline & rs9461799 & $32,797,507$ & 0.447 & 0.888 & 0.443 & 0.202 & \\
\hline & rs|3|99787 & $32,8 \mid 3,254$ & 0.449 & 0.882 & 0.436 & 0.209 & \\
\hline & rsI0807II3 & $32,830,164$ & 0.462 & 0.890 & $0.47 I$ & 0.209 & \\
\hline & rs77565I6 & $32,831,895$ & 0.462 & 0.890 & $0.47 I$ & 0.209 & \\
\hline & rs230I27I & $32,833,|7|$ & 0.413 & 0.856 & 0.427 & $0.25 \mathrm{I}$ & \\
\hline & rs7453920 & $32,837,990$ & 0.413 & 0.856 & 0.425 & 0.250 & \\
\hline & rs6903।30 & $32,840,188$ & 0.495 & 0.820 & 0.369 & 0.286 & \\
\hline & rs6901084 & $32,844,9 \mid 4$ & 0.469 & 0.890 & 0.432 & 0.194 & \\
\hline \multirow[t]{5}{*}{24} & rs946884I & $30,933,266$ & 0.076 & 0.570 & 0.146 & 0.504 & 0.0007 \\
\hline & rs775652I & $30,956,232$ & 0.178 & 0.888 & 0.360 & 0.175 & \\
\hline & rs3873334 & $31,004,126$ & 0.167 & 0.929 & 0.336 & 0.107 & \\
\hline & rs|269794| & $3|, 0| 2,693$ & 0.152 & 0.928 & 0.302 & 0.104 & \\
\hline & rs3757340 & $31,029,86 \mid$ & 0.270 & 0.554 & 0.307 & 0.644 & \\
\hline \multirow[t]{3}{*}{183} & rs3868542 & $31,253,818$ & 0.370 & 0.772 & 0.366 & 0.360 & 0.0001 \\
\hline & rs887464 & $31,253,899$ & 0.431 & 0.709 & 0.276 & 0.402 & \\
\hline & rs4122189 & $31,275,906$ & 0.233 & 0.795 & 0.556 & 0.462 & \\
\hline
\end{tabular}

aBased on HapMap Data Release 23a (Phase II), NCBI Build 36, dbSNP Build I 26.

bquared correlation coefficient between a given SNP and its own cluster.

'The next highest squared correlation coefficient between a given SNP and any other cluster.

${ }^{d} p$-Value from I df Wald $\chi^{2}$ for association with outcome, adjusted for all other clusters in the model. 
upstream of HLA-C and those in Cluster 5 immediately downstream of the gene, it is likely that both clusters jointly capture the variation in the HLA-C locus. The SNPs in Cluster 20 are located in the MHC class II region. Interestingly, three of the nine SNPs in Cluster 20 are located within $H L A-D Q B 2$, and the remaining six are situated in regions directly flanking HLA-DQB2, implying that Cluster 20 may represent the DQB2 locus. Also shown in Table 4, all SNPs within each cluster are highly correlated, and each cluster is of relatively stable fit.

\section{Discussion}

Traditionally, investigators examining gene regions or specific candidate genes might genotype hundreds of SNPs, possibly perform tag SNP selection, and test each SNP for association with disease or disease-related traits. Unfortunately, this approach necessitates multiple test correction, resulting in a significant reduction in power. PC analysis has been suggested as an exploratory approach that parses the information contained in a large number of correlated SNPs into a smaller number of orthogonal PCs that can be analyzed for association instead of individual SNPs [3,4]. A significant omnibus test of PCs indicates statistical association between a given region, as represented by the SNPs genotyped, and disease outcomes. However, PCA cannot be used to identify the specific SNPs contributing to the association, and therefore still requires testing of individual variants, to isolate the specific $\operatorname{SNP}(\mathrm{s})$ contributing to the association. We introduce a PC-based clustering method that retains many of the favorable attributes of $\mathrm{PC}$ regression, but allows for identification of the subset of SNPs contributing to the evidence for association, which reduces the multiple testing burden. We compared the traditional PC approach to the PC-clustering method using the NARAC data, and demonstrate that PCclustering identifies variants in the 3.2-Mb MHC region contributing to RA risk and variation in RA-related traits.

While traditional PC analysis makes it possible to analyze only the subset of PCs that represent most of the variation in a candidate region, PCs still represent linear combinations of all SNPs in the data set, which makes interpretation of significant PCs difficult. Upon inspection of the 29 PCs from the full model found to be significantly associated with RA status, we found the 822 eigenvector loadings on these PCs to range from -0.148 to 0.145 , with most hovering close to 0 . Thus, we were only able to infer from PC analysis that variation in the MHC region, as represented by these 822 SNPs, is strongly associated with RA risk. Additional interpretation of the specific SNP(s) driving significant associations between PCs and phenotypes can only be achieved by testing all 822 SNPs individually for association. In contrast, the PC-based clustering algorithm we employed reduced 822 SNPs to 188 discernable SNP clusters that also accounted for $80 \%$ of the regional variation. The clusters, which are subsets of the 822 SNPs analyzed, allow unique identification of those SNPs that may contribute to the evidence for association. For example, of the 24 SNP clusters associated with RA status, Cluster 1 and Cluster 23 were found to be the most significant. Cluster 1 represents a distinct set of SNPs covering $\sim 883 \mathrm{~kb}$ of the $3.2-\mathrm{Mb}$ region examined, while Cluster 24 covers a non-overlapping region of $\sim 295 \mathrm{~kb}$. While Cluster 1 represents SNPs flanking HLA-C and HLA-B, Cluster 23 comprises SNPs surrounding the HLA-DRA, HLA-DRB5, and HLA-DRB1 loci. In fact, rs3099844 and rs2857595 found in Cluster 1 were previously identified by Lee et al. [5] as belonging to a haplotype associated with anti-CCP positive RA, which $98 \%$ of cases in the present study were. Additionally, rs2395175 in Cluster 23 ranked among the top ten SNPs for association with $\mathrm{RA}$ in a recent genome-wide association study by Plenge et al. [8].

The clustering algorithm also identified 36 SNP clusters found to be associated with variation in RFUW among RA cases. The most significant associations included Clusters 2, 5, 20, 24, and 183. Clusters 2, 5, 24, and 183 are composed of SNPs located in the chromosomal region between $H L A-A$ and $H L A-C$, with Clusters 2 and 5 capturing the specific variation in and around HLA-C. Interestingly, Yen et al. demonstrated that $H L A-C$ alleles may modulate the pattern of RA progression [10]. Moreover, Lee et al. found rs887464 in Cluster 183 to be associated with RA affection [5]. Cluster 20, composed of nine SNPs, represents variants located within and proximal to HLA-DQB2. Previous examination of genes in the MHC class II region, conditional on the HLA-DRB loci, has shown the HLA-DQB2 locus to have a vital role in RA $[11,12]$. As RA is heterogeneous in terms of the progression of joint destruction [13], further examination of the SNPs in these clusters may provide information regarding genetic determinants of RA progression or symptom severity.

While our PC-based clustering method offers the interpretability a traditional PC approach lacks, there are other issues to be considered. First, we required more clusters than PCs to satisfy the $80 \%$ explained-variance threshold, which increased the degrees of freedom utilized for the omnibus test of association. The additional degrees of freedom usually results in reduced power to detect global association compared to the traditional PC approach. This may be due to the fact that while PCs are orthogonal, or independent, cluster components formed by the clustering algorithm are oblique. At each iteration, $\mathrm{PC}_{1}$ and $\mathrm{PC}_{2}$ are computed 
from a distinct set of SNPs that have been assigned to a given cluster, such that the first PC of one cluster may be correlated with the first PC of another cluster. Thus, although each SNP is assigned to the cluster with which it has the highest squared correlation, all SNPs share some degree of correlation with the other clusters they were not assigned to. This underlying correlation among clusters may be indicative of the correlation pattern among SNPs, although not necessarily haplotype blocks, and thus better reflect the true relationship of the variants within the MHC candidate region, but may also result in slightly reduced power to detect association.

\section{Conclusion}

Both traditional PC and PC-based clustering methods indicate the MHC gene region is significantly associated with RA and RFUW. However, traditional PCA is unable to highlight which SNPs contributed to this association. In contrast, the PC-based clustering method maintains many of the virtues of the traditional PC approach, but has the advantage of isolating the SNP(s) contributing to evidence for association. Therefore, the PC-based clustering method may be a better approach to testing multiple variant associations with phenotypes of interest.

\section{List of abbreviations used}

Anti-CCP: Anti-cyclic citrullinated peptide; GAW16: Genetic Analysis Workshop 16; MAF: Minor allele frequency; MHC: Major histocompatability complex; NARAC: North American Rheumatoid Arthritis Consortium; PCA: Principal-components analysis; RA: Rheumatoid arthritis; RFUW: Rheumatoid factor IgM; SNP: Single-nucleotide polymorphism.

\section{Competing interests}

The authors declare that they have no competing interests.

\section{Authors' contributions}

MHB conceived of the study, analyzed the data, and drafted the manuscript. RMW advised on the manuscript. Both authors read and approved the final manuscript.

\section{Acknowledgements}

The authors thank John Morrison for technical assistance in accessing the GAWI 6 data and the NARAC investigators for contributing their data to GAWI6. MHB was supported by a USC training grant in the cellular, molecular and biochemical sciences.

This article has been published as part of BMC Proceedings Volume 3 Supplement 7, 2009: Genetic Analysis Workshop 16. The full contents of the supplement are available online at http://www.biomedcentral.com/ $|753-656| / 3$ ? issue=S7.

\section{References}

I. Vermeulen SHHM, Den Heijer M, Sham P and Knight J: Application of multi-locus analytical methods to identify interacting loci in case-control studies. Ann Hum Genet 2007, 71:689-700.

2. Heidema AG, Boer JMA, Nagelkerke N, Mariman ECM, Van Der ADL and Feskens EJM: The challenge for genetic epidemiologists: how to analyze large numbers of SNPs in relation to complex diseases. BMC Genet 2006, 7:23.

3. Gauderman JW, Murcray C, Gilliland F and Conti D: Testing association between disease and multiple SNPs in a candidate gene. Genet Epidemiol 2007, 31:383-395.

4. Wang $\mathrm{K}$ and Abbott $\mathrm{D}$ : $\mathbf{A}$ principal components regression approach to multilocus genetic association studies. Genet Epidemiol 2008, 32:108-118.

5. Lee HS, Lee AT, Criswell LA Seldin MF, Amos Cl, Carulli JP Navarrete C, Remmers EF, Kastner DL, Plenge RM, Li W and Gregersen PK: Several regions in the major histocompatibility complex confer risk for anti-CCP-antibody positive rheumatoid arthritis, independent of the DRBI locus. Mol Med 2008, I 4:293-300

6. Irigoyen P, Lee AT, Wener MH, Li W, Kern M, Batliwalla F, Lum RF, Massarotti E, Weisman M, Bombardier C, Remmers EF, Kastner DL, Seldin MF, Criswell LA and Gregersen PK: Regulation of anticyclic citrullinated peptide antibodies in rheumatoid arthritis: contrasting effects of HLA-DR3 and the shared epitope alleles. Arthritis Rheum 2005, 52:38I3-3818.

7. van Gaalen FA, van Aken J, Huizinga TW, Schreuder GM, Breedveld FC, Zanelli E, van Venrooij WJ, Verweij CL, Toes RE and de Vries RR: Association between HLA class II genes and autoantibodies to cyclic citrullinated peptides (CCPs) influences the severity of rheumatoid arthritis. Arthritis Rheum 2004, 50:2113-2I21.

8. Plenge RM, Cotsapas C, Davies L, Price AL, de Bakker PI, Maller I Pe'er I, Burtt NP, Blumenstiel B, DeFelice M, Parkin M, Barry R, Winslow W, Healy C, Graham RR, Neale BM, Izmailova E, Roubenoff R, Parker AN, Glass R, Karlson EW, Maher N, Hafler DA, Lee DM, Seldin MF, Remmers EF, Lee AT, Padyukov L, Alfredsson L, Coblyn J, Weinblatt ME, Gabriel SB, Purcell S, Klareskog L, Gregersen PK, Shadick NA, Daly MJ and Altshuler D: Two independent alleles at $6 \mathrm{q} 23$ associated with risk of rheumatoid arthritis. Nat Genet 2007, 39:|477-|482.

9. Harris CW and Kaiser HF: Oblique factor analytic solutions by orthogonal transformations. Psychometrika 1964, 29:347-362.

10. Yen JH, Moore BE, Nakajima T, Scholl D, Schaid DJ, Weyand CM and Goronzy JJ: Major histocompatibility complex class I-recognizing receptors are disease risk genes in rheumatoid arthritis. J Exp Med 200I, I93: I I59-I I67.

I I. Shiina T, Inoko H and Kulski JK: An update of the HLA genomic region, locus information and disease associations: 2004. Tissue Antigens 2004, 64:631-639.

12. Kochi Y, Yamada R, Kobayashi K, Takahashi A, Suzuki A, Sekine A Mabuchi A, Akiyama F, Tsunoda T, Nakamura $Y$ and Yamamoto K: Analysis of single-nucleotide polymorphisms in Japanese rheumatoid arthritis patients shows additional susceptibility markers besides the classic shared epitope susceptibility sequences. Arthritis Rheum 2004, 50:63-7I.

13. Weyand CM, Klimiuk PA and Goronzy J]: Heterogeneity of rheumatoid arthritis: from phenotypes to genotypes. Springer Semin Immunopathol 1998, 20:5-22.

Publish with BioMed Central and every scientist can read your work free of charge

"BioMed Central will be the most significant development for disseminating the results of biomedical research in our lifetime. "

Sir Paul Nurse, Cancer Research UK

Your research papers will be:

- available free of charge to the entire biomedical community

- peer reviewed and published immediately upon acceptance

- cited in PubMed and archived on PubMed Central

- yours - you keep the copyright
BioMedcentral 